\title{
Video-Assisted Thoracic Surgery for Residual Aneurysm after Total Arch Replacement
}

\author{
Kayo Sugiyama ${ }^{1}$, Hirotaka Watanuki ${ }^{1}$, Yasuhiro Futamura ${ }^{1}$, Masaho Okada ${ }^{1}$, Hiroki Numanami ${ }^{2}$, \\ Masayuki Yamaji², Satoshi Makino', Katsuhiko Matsuyama1
}

${ }^{1}$ Department of Cardiac Surgery, Aichi Medical University, Nagakute, Japan

${ }^{2}$ Department of Thoracic Surgery, Aichi Medical University, Nagakute, Japan

Email: kayotaro3@gmail.com

How to cite this paper: Sugiyama, K., Watanuki, H., Futamura, Y., Okada, M., Numanami, H., Yamaji, M., Makino, S. and Matsuyama, K. (2021) Video-Assisted Thoracic Surgery for Residual Aneurysm after Total Arch Replacement. Open Journal of Thoracic Surgery, 11, 25-30.

https://doi.org/10.4236/ojts.2021.111004

Received: January 12, 2021

Accepted: March 12, 2021

Published: March 15, 2021

Copyright (อ 2021 by author(s) and Scientific Research Publishing Inc. This work is licensed under the Creative Commons Attribution International License (CC BY 4.0).

http://creativecommons.org/licenses/by/4.0/

\begin{abstract}
Background: Residual aneurysms after graft replacement are rare, but they can be detrimental if they are saccular and large. The etiology of residual aneurysms remains unknown, and their management is controversial. One treatment option is late open surgical conversion; however, postoperative respiratory complications resulting from the dissection of pleural adhesions, which is frequently necessary with this approach, are often unavoidable. Case presentation: Herein, we report a case of open surgical repair of a residual distal aortic arch aneurysm that occurred after total arch replacement and thoracic endovascular aortic repair. Contrast-enhanced magnetic resonance imaging was not possible in this case due to the patient's severe renal dysfunction; however, contrast-enhanced computed tomography using minimal contrast did not detect remarkable leakage through the graft or stent graft into the aneurysm. Late open surgical conversion using video-assisted thoracic surgery was performed by thoracic surgeons, and the adhesion between the aortic wall and the lung was safely and effectively dissected. Because there was no significant pulsation or evidence of feeding arteries in the aortic wall, the aortic wall was opened carefully. No bleeding or backflow from any branch arteries into the aneurysm was noted, so the aortic wall was ligated with continuous sutures. The patient recovered without experiencing any major complications. Conclusions: This case report demonstrates that video-assisted thoracic surgery is safe and effective for late open conversion in cases of residual aneurysm; furthermore, this case suggests that video-assisted thoracic surgery may be particularly beneficial for the dissection of adhesions between the aortic wall and lung in these cases.
\end{abstract}

\section{Keywords}

Late Open Surgical Conversion, Total Arch Replacement, Video-Assisted Thoracic Surgery 


\section{Introduction}

The etiology of residual aneurysms after graft replacement is still unclear. Because problems related to residual aneurysms are rare, their management is controversial. However, they can be fatal if they rupture. Most cases of residual aneurysm can be treated with endovascular aortic repair [1] [2]; however, late open surgical conversion is sometimes unavoidable [3] [4].

Some methods have been proposed as viable alternatives to late open surgical conversion; however, these procedures are not completely reliable for residual aneurysms of unknown etiology [5] [6]. Open surgery necessitates thoracotomy and can cause lung injury during dissection of pleural adhesions. Video-assisted thoracic surgery (VATS) is reportedly less invasive for open surgical repair and effective for the dissection of adhesions between the aorta and the lung [7]. Herein, we report a case of successfully open repair using VATS for residual aneurysm after aortic arch repair.

\section{Case Report}

A 75-year-old male patient with dilatation of a distal arch aneurysm after open surgical repair was referred to us for surgical treatment. Twelve years prior, the patient was treated for hypertension at a local hospital and had undergone graft replacement for an abdominal aortic aneurysm. Eight years later, he developed an acute Stanford type B aortic dissection and underwent a graft replacement for the distal aortic arch aneurysm using a $26-\mathrm{mm}$ four-branched Triplex artificial graft (Terumo Corporation, Tokyo, Japan) and a $31-60$ mm Frozenix J Graft open stent graft (Japan Lifeline, Tokyo, Japan) [Figure 1(a)]. One month later, the patient also underwent thoracic endovascular aortic repair for dilatation of the descending aorta using a 36-32-200 mm and 32-32-80 mm TX2 (Cook Inc., Bloomington, IN). Postoperative computed tomography showed only a type II endoleak via intercostal arteries, and the patient did well without aortic dilatation during a 4-year follow-up period. However, upon follow-up evaluation in our department via plain computed tomography, a slight dilatation of the distal aortic arch was detected. Since the aneurysm was a large and saccular type, we considered the aneurysm to be at high risk for rupture and untreatable via endovascular aortic repair due to the unknown etiology of the dilatation.

The patient's preoperative laboratory evaluation indicated mild anemia and severe chronic kidney disease. Relevant results from that testing include the following: white blood cell count, $4.2 \times 10^{3} / \mu \mathrm{L}$; C-reactive protein level, $0.1 \mathrm{mg} / \mathrm{dL}$; creatinine level, $2.6 \mathrm{mg} / \mathrm{dL}$; estimated glomerular filtration rate, $20 \mathrm{~mL} / \mathrm{min} / 1.73$ $\mathrm{m}^{2}$; and hemoglobin level, $10.7 \mathrm{~g} / \mathrm{dL}$. Preoperative computed tomography using minimal contrast did not detect either inflow or outflow branch vessels through the aneurysm, nor did it find remarkable leakage through the graft or stent graft into the aneurysm; in fact, the only finding was a slight enhancement of the aortic wall [Figure 1(b)]. Therefore, relining of the previous stent graft was thought to be an ineffective strategy. 


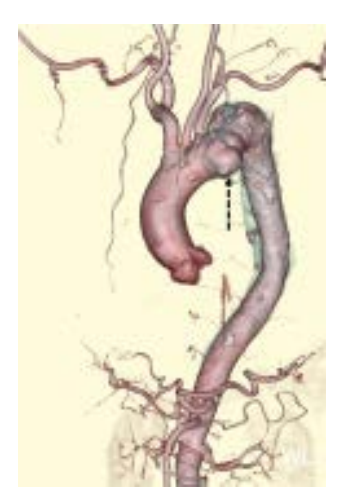

(a)

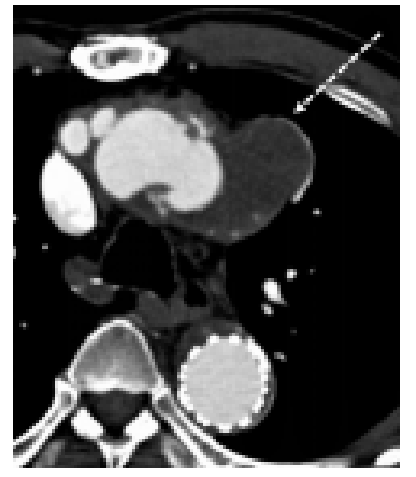

(b)

Figure 1. (a) Computed tomography scan taken before the last surgery showing a saccular aneurysm (black arrow) in the aortic arch; (b) Preoperative computed tomography showing a residual saccular aneurysm (white arrow) in the aortic arch with no apparent endoleak or active bleeding.

Considering the patient's comorbidities, late open surgical conversion for a residual saccular distal arch aneurysm was scheduled. Because severe adhesion was presupposed in this case, a less-invasive VATS procedure was chosen. Using VATS, dissection of an adhesion between the aorta and lung was safely performed by thoracic surgeons [Figure 2(a), Figure 2(b)]. Because the distal arch aneurysm was not pulsatile and could not be palpated, and because no feeding arteries were detected around the aortic wall [Figure 2(b)], the aneurysm was carefully opened. No bleeding or backflow from any branch vessels into the aneurysm occurred [Figure 2(c)], and after clipping the vasa vasorum with a vascular clip, the aneurysmal wall was ligated with continuous sutures [Figure 2(d)]. The patient recovered without any complications. The patient has been doing well without major adverse aortic events or aortic dilatation as of 6 months after surgery.

The patient provided permission to publish the features of his case. The identity of the patient has been protected. This case report was approved by the Institutional Review Board of Aichi Medical University Hospital.

\section{Discussion}

The etiology of residual aneurysms after graft replacement is still unclear. These may be due to the presence of an unrecognized endoleak that is below the resolution of current imaging methods, transmission of pressure through the thrombus or stent graft, or increased permeability from the covering material of the graft itself [8]. Untreated endotension may lead to enlargement and subsequent rupture of the aneurysmal sac [1]. It is difficult to confirm this diagnosis, though it can be fatal if rupture occurs. The etiology of residual saccular aneurysms after open graft replacement is still unknown; however, an unrecognized endoleak might be related to its rupture. Because problems related to residual aneurysms after open graft replacement are rare, their management is controversial. 


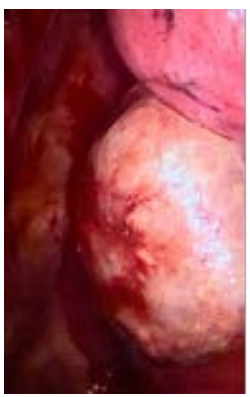

(a)

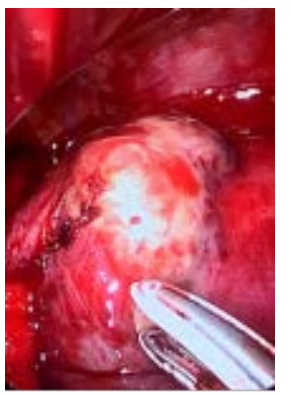

(b)

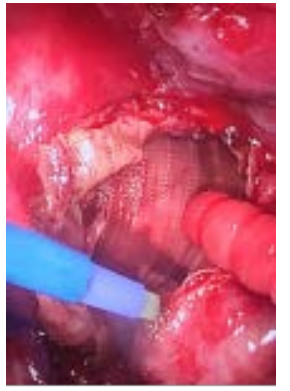

(c)

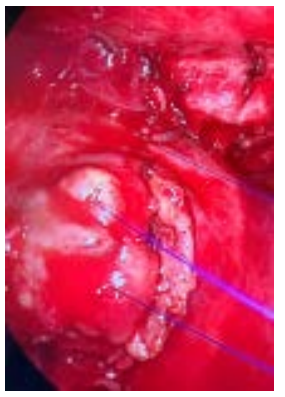

(d)

Figure 2. (a) Intraoperative view showing dissection of the adhesion between the aorta and lung using VATS; (b) Intraoperative view showing the pulse-less aneurysm in the aortic arch; (c) Intraoperative view showing the opened aneurysm with no apparent endoleak or active bleeding; (d) Intraoperative view showing the ligated aneurysm.

Endovascular redo stent graft placement has reportedly been effective in inducing sac shrinkage in patients with endotension [1], and most cases can be treated by endovascular aortic repair [1] [2]; however, late open surgical conversion is sometimes required [3] [4]. Late open surgical conversion after thoracic endovascular aortic repair (TEVAR) is reportedly observed in $2 \%-8 \%$ of cases [9] [10]. A progressive increase in aneurysm size without identifiable endoleak is one of the major limitations of endovascular treatment [10]. Similarly, the present case showed no remarkable leakage through the graft or stent graft into the aneurysm except for a slight enhancement of the aortic wall. Because of progressive aneurysm enlargement, a surgical conversion is sometimes necessary to remove the stent-graft and reconstruct the aorta. Canaud et al. reported 14 cases that required open surgical conversion after TEVAR including 2 cases that underwent surgery because of aneurysm enlargement without identifiable endoleak [10]. Nano et al. treated endotension with aortic sac evacuation and wrapping of the endograft [5]. Alternative approaches for progressive aneurysm enlargement include puncture of the aneurysm sac and removal of its contents [6] or fenestration of the aneurysm sac to depressurize it and prevent its continued expansion due to the accumulation of material and/or fluid [6]. When an aneurysm is excluded from the circulation, its wall is no longer exposed to systemic pressure. However, these procedures cannot be completely reliable for residual aneurysm of unknown etiology. In the present case, the aneurysm was opened and ligated. About the morphological change and size of the aneurysm, careful follow-up is required.

In late open surgical conversion after TEVAR, postoperative respiratory complications are frequently unavoidable after the dissection of pleural adhesions that is necessary for extensive thoracic exposure [9]. Pleural adhesions due to the aortic inflammatory response are not uncommon after TEVAR, and a cautious aortic exposure limiting as much as possible pleural and lung lesions is advocated to reduce postoperative respiratory complications [9]. VATS for thoracic aortic aneurysm is also beneficial because it is less invasive than open surgery [7]. In cases after EVAR for abdominal aortic aneurysm, successful laparoscopic 
fenestration of the aneurysm sac with removal of all thrombotic material has been reported [5] [6] [8]; however, there have been few reports describing the usefulness of VATS as late open conversion after TEVAR. Although the VATS procedure is widely disseminated in the field of thoracic surgery, cardiac surgeons are not familiar with this. Because there is no guarantee that simple ligation of the residual aneurysm without remarkable leakage is also effective in the late phase, further reports regarding late open surgical conversion after thoracic aortic surgery are warranted.

\section{Conclusion}

In this case, VATS enabled us to perform safe and successful aneurysm repair despite the presence of severe pleural adhesion. For thoracic aortic aneurysms that cannot be treated with endovascular treatment, VATS will be a more useful procedure than open surgery.

\section{Acknowledgements}

We thank the Honyaku Center staff for reviewing and editing the manuscript. We also thank our colleagues for their helpful comments.

\section{Conflicts of Interest}

The authors declare no conflicts of interest regarding the publication of this paper.

\section{References}

[1] Zimpfer, D., Schoder, M., Gottardi, R., Lammer, J., Wolner, E., Grimm, M. and Czerny, M. (2007) Treatment of Type V Endoleaks by Endovascular Redo Stent-Graft Placement. The Annals of Thoracic Surgery, 83, 664-666. https://doi.org/10.1016/j.athoracsur.2005.06.054

[2] Kostun, Z. and Mehta, M. (2107) TEVAR and Reintervention: How to Manage Endoleaks and False Lumen Perfusion. Endovascular Today, 16, 60-62.

[3] Bonardelli, S., Nodari, F., De Lucia, M., Botteri, E., Benenati, A. and Cervi, E. (2018) Late Open Conversion after Endovascular Repair of Abdominal Aneurysm Failure: Better and Easier Option than Complex Endovascular Treatment. JRSM Cardiovascular Disease, 7, 1-6. https://doi.org/10.1177/2048004017752835

[4] Goudeketting, S.R., Jin, P.F.K., Ünlü, Ç. and Vries, J.P.P.M. (2019) Systematic Review and Meta-Analysis of Elective and Urgent Late Open Conversion after Failed Endovascular Aneurysm Repair. Journal of Vascular Surgery, 70, 615-628. https://doi.org/10.1016/j.jvs.2018.11.022

[5] Nano, G., Dalainas, I., Bianchi, P.G., Gotti, R., Casana, R., Malacrida, G. and Tealdi, D.G. (2006) Sac Enlargement due to Seroma after Endovascular Abdominal Aortic Aneurysm Repair with the Endologix PowerLink Device. Journal of Vascular Surgery, 43, 169-171. https://doi.org/10.1016/j.jvs.2005.09.011

[6] Nes, J.G.H., Hendriks, J.M., Tseng, L.N.L., Dijk, L.C. and Sambeek, M.R.H.M. (2005) Endoscopic Aneurysm Sac Fenestration as a Treatment Option for Growing Aneurysms due to Type II Endoleak or Endotension. Journal of Endovascular Therapy, 12, 430-434. https://doi.org/10.1583/05-1541R.1 
[7] Taylor, L.J., Adesoye, T. and Maloney, J.D. (2016) Vide-Assisted Thoracic Surgery for Cancer after Thoracic Aortic Aneurysm Repair. Asian Cardiovascular and Thoracic Annals, 24, 201-203. https://doi.org/10.1177/0218492315610264

[8] Cerna, M., Köcher, M., Utikal, P. and Bachleda, P. (2009) Endotension after Endovascular Treatment of Abdominal Aortic Aneurysm: Percutaneous Treatment. Journal of Vascular Surgery, 50, 648-651. https://doi.org/10.1016/j.jvs.2009.04.044

[9] Rinaldi, E., Kahlberg, A., Carta, N., Mascia, D., Bertoglio, L. and Chiesa, R. (2020) Late Open Conversion Following Failure of EVAR and TEVAR: "State of the Art". Cardiovasc Intervent Radiol, 43, 1855-1864.

[10] Canaud, L., Alric, P., Gandet, T., Ozdemir, B.A., Albat, B. and Marty-Ane, C. (2013) Open Surgical Secondary Procedures after Thoracic Endovascular Aortic Repair. European Journal of Vascular and Endovascular Surgery, 46, 667-674.

https://doi.org/10.1016/j.ejvs.2013.08.022 